International Journal of Modern Physics D

(C) World Scientific Publishing Company

\title{
LIGHT SUPERCONDUCTING STRINGS IN THE GALAXY*
}

\author{
Francesc Ferrer and Tanmay Vachaspati \\ CERCA, Department of Physics, Case Western Reserve University, \\ 10900 Euclid Avenue, Cleveland, OH 44106-7079, USA
}

\begin{abstract}
Observations of the Milky Way by the SPI/INTEGRAL satellite have confirmed the presence of a strong $511 \mathrm{KeV}$ gamma-ray line emission from the bulge, which require an intense source of positrons in the galactic center. These observations are hard to account for by conventional astrophysical scenarios, whereas other proposals, such as light DM, face stringent constraints from the diffuse gamma-ray background. Here we suggest that light superconducting strings could be the source of the observed $511 \mathrm{KeV}$ emission. The associated particle physics, at the $\sim 1 \mathrm{TeV}$ scale, is within reach of planned accelerator experiments, while the distinguishing spatial distribution, proportional to the galactic magnetic field, could be mapped by SPI or by future, more sensitive, satellite missions.
\end{abstract}

Keywords: positrons, strings, galaxy

\section{Positron Sources in the Galaxy}

The problem of the birth, propagation and annihilation of positrons in the Galaxy has been a major topic of astrophysical investigation, since the first detection ${ }^{1}$ of the $511 \mathrm{KeV}$ gamma-ray line signature. The SPI instrument on-board ESA's INTEGRAL satellite has established the presence of a diffuse source of positrons in the Galactic Center $(\mathrm{GC})^{2}$. The observed photon flux of

$$
9.9_{-2.1}^{+4.7} \times 10^{-4} \mathrm{~cm}^{-2} \mathrm{~s}^{-1}
$$

with a line-width of about $3 \mathrm{KeV}$ is in good agreement with previous measurements ${ }^{3}$.

For the spatial distribution of the $511 \mathrm{KeV}$ line component, the mapping results point to an intense bulge emission, better explained by an extended distribution than by a point source. Assuming a Gaussian spatial distribution for the flux, a full-width at half maximum of $9^{\circ}$ is indicated. The disk component has been either absent, or weakly detected in the initial results.

The origin of these Galactic positrons remains a mystery. Several scenarios involving astrophysical sources have been proposed, including neutron stars or black holes, massive stars, supernovae, hypernovae, gamma ray bursts or cosmic rays ${ }^{4}$. However, the fraction of positrons produced in such processes is uncertain, and it is unclear that the positrons could fill the whole bulge.

*Talk given by T. Vachaspati at the workshop From Quantum to Cosmos: Fundamental Physics Research in Space, Washington, D. C., May 2006 
Alternatively, mechanisms associated with the Dark Matter (DM) at the GC have been put forward. If DM is constituted by a light ( $\mathrm{MeV}$ ) scalar, its decay or annihilation could account for the observed signal ${ }^{5}$.

The positrons should be injected at non-relativistic energies so that the associated bremsstrahlung emission does not violate the COMPTEL and EGRET measurements of diffuse radiation from the Galactic Center ${ }^{6}$. For the DM scenario, this implies that the DM particles should be lighter than $\sim 20-30 \mathrm{MeV}$. Moreover, inflight annihilation of positrons would also overproduce gamma-rays unless the positrons are injected at energies below $\sim 3 \mathrm{MeV}^{7}$, thus disfavoring some of the scenarios in ${ }^{4}$ and $^{5}$.

We will discuss here the possibility, proposed in $^{8}$, that a network of light superconducting strings ${ }^{9}$ occurring in particle physics just beyond the standard model could be a source of the galactic positrons. This scenario predicts a characteristic positron distribution that could be used to distinguish this source from the other possibilities.

Assuming that a tangle of superconducting strings exists in the Milky Way, then the strings are frozen in the plasma as long as the radius of curvature is larger than a certain critical length scale. If the curvature radius is smaller, the string tension wins over the plasma forces and the string moves with respect to the magnetized plasma. During the string motion, the loop will cut across the Milky Way magnetic field, generating current as given by Faraday's law of induction.

The current is composed of zero modes of charged particles, including positrons, propagating along the string. The external magnetic field shifts the modes of the charge carriers into the bulk and modifies the dispersion relation dramatically so that their energy remains below the threshold for expulsion ${ }^{10}$ (which for the positron zero modes is $511 \mathrm{KeV}$ ). An additional perturbation, like inhomogeneities in the magnetic field, string motion and curvature, or scattering by counter-propagating particles ${ }^{11}$, ejects the zero modes at the threshold of $511 \mathrm{KeV}$. The ejected positrons will annihilate with the ambient electrons, thus emitting $511 \mathrm{KeV}$ gamma rays.

\section{Light superconducting strings in the Galaxy}

The amount of positrons injected in the Milky Way, will depend on how many strings are injecting positrons per unit volume and on the output rate of positrons per unit length of string. Let us first estimate the density of strings in the Galaxy.

The strings, being superconducting, can sustain currents which couple the dynamics of the string network to the Milky Way plasma. The density of strings will, thus, depend both on the properties of the string (like its tension, $\mu$, radius of curvature, $R$, and the intensity of the current being carried, $J$ ) and of the plasma (like its density, $\rho$ ).

The dynamics is determined by comparing the force due to string tension, $F_{s}$, to the plasma drag force, $F_{\mathrm{d}}$. The analysis ${ }^{12}$ shows that there is a critical radius of 
curvature,

$$
R_{c} \sim \frac{\mu}{\sqrt{\rho} J}
$$

such that the plasma drag can not check the force due to the string tension when $R<R_{c}$, and the strings move at relativistic speeds. String loops will then emit electromagnetic radiation and eventually dissipate.

On the other hand, less curved strings, i. e. for $R>R_{c}$, accelerate under their own tension until they reach a terminal velocity

$$
v_{\text {term }} \sim \frac{\mu}{\sqrt{\rho} J R} .
$$

In a turbulent plasma, such as in our Milky Way, there is another length scale of interest, called $R_{*}\left(R_{*}>R_{c}\right)$, even when the string motion is overdamped. For $R>R_{*}$, the terminal speed of the strings is small compared to the turbulence speed of the plasma and the strings are carried along with the plasma. As the strings follow the plasma flow, they get more entangled due to turbulent eddies, and the strings get more curved until the curvature radius drops below $R_{*}$. Then the string velocity is large compared to the plasma velocity, and, hence, the strings break away from the turbulent flow. Therefore, $R_{*}$ is the smallest scale at which the string network follows the plasma flow. For $R_{*}>R>R_{c}$, the string motion is over-damped but independent of the turbulent flow. Hence, string curvature on these scales is not generated by the turbulence, and we can estimate the length density of strings in the plasma as $\rho_{l} \sim 1 / R_{*}^{2}$. The scale $R_{*}$ at which the terminal velocity (3) equals the turbulent velocity of the plasma, $v_{*}$, is given by ${ }^{13}$ :

$$
R_{*} \sim l\left(\sqrt{\frac{\mu}{\rho}} \frac{1}{e \kappa v_{l} l}\right)^{4 / 5}, \quad v_{*} \sim v_{l}\left(\sqrt{\frac{\mu}{\rho}} \frac{1}{e \kappa v_{l} l}\right)^{1 / 5},
$$

where, for convenience, the dimensionless parameter $\kappa$ has been introduced via $J \equiv \kappa e \sqrt{\mu}$.

\section{Particle emission by superconducting strings}

When a string of typical length $R_{*}$ moves at a velocity $v_{*}$ with respect to the Milky Way plasma, it cuts across the galactic magnetic field lines and a current is generated on the string as described by Faraday's law of induction.

The same magnetic field that creates the current, changes the dispersion relation of the zero modes. In the absence of the external magnetic field, the zero modes behave as massless particles, with a linear dispersion relation. The current could increase indefinitely as the zero modes gain momentum. However, the presence of the external magnetic field changes the dispersion relation of the zero modes dramatically ${ }^{10}$ and it can be approximated by:

$$
\omega_{k}=m_{e^{+}} \tanh \left(\frac{k}{k_{*}}\right)
$$


where $k_{*}$ is a parameter that depends on the magnetic field. Consequently, the current on the string saturates at $J_{\max }=e \mathrm{~m}_{e^{+}}$, and the positron zero modes have energies approaching from below the threshold for expulsion, $511 \mathrm{KeV}$.

The string will, in general, carry additional zero modes corresponding to other heavier particles, say heavy quarks. Then, the total current in the string, entering the network dynamics in Eq. (4), will be determined by the heavier particles. The positron current, though, will still be bounded to be below $511 \mathrm{KeV}$.

A given charge carrier can, in principle, leave the string once it has enough energy. The escape is triggered by several factors such as string motion and curvature or scattering by counter-propagating particles ${ }^{11}$ ( $u$ quarks for electroweak strings ${ }^{8}$ ), but in any case, the positrons will be emitted at their threshold energy, $511 \mathrm{KeV}$.

As a piece of string of length $R_{*}$ cuts through a magnetic field $B$, it will produce electrons or positrons, with equal likelihood, at the rate

$$
\frac{d N}{d t} \sim e v_{*} B R_{*}
$$

In a volume $V=4 \pi L^{3} / 3$, there are of order $L^{3} / R_{*}^{3}$ such pieces of string and hence the rate of particle production in the entire volume is:

$$
\frac{d N_{\mathrm{V}}}{d t} \sim e v_{*} B \frac{L^{3}}{R_{*}^{2}}
$$

The current in the positrons will grow at first, but then saturate at $511 \mathrm{KeV}$. After that, further motion of the string across the galactic magnetic field will generate positrons that leave the string. So $N_{V}$ is also the number of positrons being produced in the volume $V$ which we denote by $N_{+}$. Inserting Eqs. (4) in (7) we get:

$$
\frac{d N_{+}}{d t} \sim 10^{42} B_{3} \kappa^{7 / 5} V_{1} \mu_{1}^{-7 / 10} \rho_{\mathrm{gc}}^{7 / 10} v_{l, 6}^{12 / 5} l_{100}^{-3 / 5} \mathrm{~s}^{-1},
$$

where we have introduced the parameters describing the plasma of the spherical region of radius $1 \mathrm{kpc}$ around the galactic center, $\rho \sim 6 \cdot 10^{-24} \rho_{\mathrm{gc}} \mathrm{gm} / \mathrm{cm}^{3}, B=$

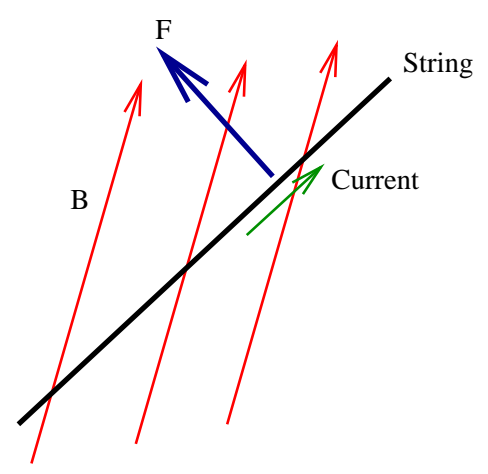

Fig. 1. The charge carriers run along the string, in the presence of a perpendicular external magnetic field. 
$B_{3} 10^{-3} \mathrm{G}, v_{l}=10^{6} v_{l, 6} \mathrm{~cm} / \mathrm{s}$ and $l=100 l_{100} \mathrm{pc}$. The string tension is given by $\mu=\mu_{1}(1 \mathrm{TeV})^{2}$.

Although the astrophysical parameters describing the galactic center are not known very accurately, assuming equipartition of plasma kinetic energy $\left(\sim \rho v_{l}^{2}\right)$ and magnetic energy $\left(\sim B^{2} / 8 \pi\right)$, with $l \sim \rho^{-1 / 3}$, we find that $v_{l, 6} \sim 100$ and $l_{100} \sim 0.1$ which boosts the estimate in Eq. (8) by $10^{5}$, yielding

$$
\frac{d N_{+}}{d t} \lesssim 10^{47} \mathrm{~s}^{-1}
$$

This should be compared with the actual positron production rate in the galactic center:

$$
\frac{d N_{+}^{o b s}}{d t} \sim 1.2 \times 10^{43} \mathrm{~s}^{-1} .
$$

Comparing Eqs. (9) and (10) we conclude that light superconducting strings are possible sources of positrons that lead to the flux of $511 \mathrm{KeV}$ gamma rays observed by the INTEGRAL collaboration.

\section{Observational signatures}

We see from Eq. (8) that a unique prediction of our scenario is that the gamma ray flux is proportional to the strength of the magnetic field in the Milky Way, with a milder dependence on the plasma density. In the disk, $B_{3} \sim 10^{-3}$, and we estimate a photon flux $\sim 10^{-6} \mathrm{~cm}^{-2} \mathrm{~S}^{-1}$ in a $16^{\circ}$ field of view as in SPI. The target sensitivity of the SPI instrument, once sufficient exposure becomes available, is $2 \times 10^{-5} \mathrm{~cm}^{-2} \mathrm{~s}^{-1}$ at $511 \mathrm{KeV}^{14}$. This threshold is somewhat above what is needed to map the emission from the disk in our scenario.

That the flux should follow the magnetic field is in marked contrast with the $\mathrm{MeV}$ DM hypothesis. There, the flux follows $\rho_{\text {DM }}^{2}$, and a signal from nearby DM dominated regions, e.g. the Sagittarius dSph galaxy, is expected ${ }^{15}$. If superconducting strings source the observed $511 \mathrm{KeV}$, however, at most, a flux of $\sim 10^{-7} \mathrm{~cm}^{-2} \mathrm{~s}^{-1}$ in the direction of Sagittarius is expected ${ }^{8}$, some three orders of magnitude fainter than the MeV DM model prediction

The strings are expected to carry additional zero modes apart from the ones corresponding to $e^{ \pm}$. We expect, thus, the presence of other currents, each saturated at the mass $m_{X}$ of the particle in vacuum, which would potentially result in the ejection of these particles also at threshold, although the presence of conserved charges might inhibit or delay some of these processes. For instance, since pion emission cannot deplete the baryonic current on the string, only at $\sim 1 \mathrm{GeV}$ energies can antiprotons be emitted, leading to another possible signature of galactic superconducting strings ${ }^{16}$.

Our scenario could also explain the excess of high-energy positrons in cosmic rays at energies around $10 \mathrm{GeV}$ detected by the HEAT balloon experiment ${ }^{17}$. Since the positron current cannot build up beyond $511 \mathrm{KeV}$ in the presence of the external magnetic field, additional heavy charged fermions would be responsible, after 
decaying or annihilating with ambient particles in the plasma, for the positrons in the $10 \mathrm{GeV}$ energy range ${ }^{8}$.

\section{What can NASA do to check these predictions}

The experimental results obtained by the SPI/INTEGRAL collaboration, have confirmed the puzzle of the positron injection in the Galactic bulge and sparked, in the few years since the publication of the first results, a plethora of possible explanations.

The distinguishing feature of our scenario is the spatial distribution tracking the magnetic field intensity. SPI will not be able to attain the sensitivity required to observe the emission from the galactic disk in our model. An improvement of roughly an order of magnitude in the sensitivity would suffice to pursue this task ${ }^{\mathrm{a}}$. This order of magnitude improvement would also test predictions from other scenarios. For instance, the signal from nearby DM clumps expected in light DM scenarios could be unveiled or, else, the models would be disproved (barring astrophysical uncertainties in the region of the clumps).

It is noteworthy, that the analysis of complementary data coming from older satellites like COMPTEL and EGRET, provides some of the most stringent constraints for all the scenarios ${ }^{6,7}$. In this respect, light superconducting strings remain a viable proposal, since the positrons are emitted at threshold, well below the $\sim 3 \mathrm{MeV}$ bounds from inflight annihilation. In should be stressed that these bounds require the knowledge of the diffuse gamma-ray flux in the galactic center to a great accuracy. With the data at hand, extrapolations of data at different energies and from different regions are necessary, which add uncertainty to the bounds. The forthcoming GLAST satellite, partially funded by NASA, is better suited to energies above $1 \mathrm{GeV}$. The task remains to get more precise data at lower energies. SPI is already contributing to this effort, but a more precise experiment would make a difference.

\section{Acknowledgments}

\section{References}

1. W. N. Johnson III, F. R. Harnden Jr. and R. C. Haymes, Astrophys. Jour. 172, L1 (1972).

2. P. Jean et al., Astron. Astrophys. 407, L55 (2003); Knödlseder et al., Astron. Astrophys. 411, L457 (2003); G. Weidenspointner et al., arXiv:astro-ph/0601673; P. Jean et al., Astron. Astrophys. 445, 579 (2006); J. Knodlseder et al., Astron. Astrophys. 441, 513 (2005); B. J. Teegarden et al., Astrophys. J. 621, 296 (2005).

3. P. A. Milne et al., New Astron. Rev. 46, 553 (2002), and references therein

4. P. A. Milne, L. S. The and M. D. Leising, arXiv:astro-ph/0104185; M. Casse et al., Astrophys. J. 602, L17 (2004); G. Bertone et al., Phys. Lett. B 636, 20 (2006) N. Prant-

${ }^{a}$ Other sources, e.g. cosmic rays, could contribute to the emission from the disk at a level that could be mapped by SPI 
zos, Astron. Astrophys. 449, 869 (2006); N. Guessoum, P. Jean and N. Prantzos, arXiv:astro-ph/0607296.

5. C. Boehm et al., Phys. Rev. Lett. 92, 101301 (2004); D. Hooper and L. T. Wang, Phys. Rev. D 70, 063506 (2004); D. H. Oaknin and A. R. Zhitnitsky, Phys. Rev. Lett. 94, 101301 (2005); S. Kasuya and M. Kawasaki, Phys. Rev. D 73, 063007 (2006); S. Kasuya and F. Takahashi, Phys. Rev. D 72, 085015 (2005).

6. J. F. Beacom, N. F. Bell and G. Bertone, Phys. Rev. Lett. 94, 171301 (2005); P. Sizun, M. Casse and S. Schanne, arXiv:astro-ph/0607374; C. Boehm and P. Uwer, arXiv:hep$\mathrm{ph} / 0606058$.

7. J. F. Beacom and H. Yuksel, arXiv:astro-ph/0512411.

8. F. Ferrer and T. Vachaspati, Phys. Rev. Lett. 95, 261302 (2005).

9. E. Witten, Nucl. Phys. B 249, 557 (1985)

10. F. Ferrer, H. Mathur, T. Vachaspati and G. D. Starkman, Phys. Rev. D 74, 025012 (2006)

11. S. M. Barr and A. M. Matheson, Phys. Lett. B 198, 146 (1987); S. M. Barr and A. M. Matheson, Phys. Rev. D 39, 412 (1989)

12. E. M. Chudnovsky, G. B. Field, D. N. Spergel and A. Vilenkin, Phys. Rev. D 34, 944 (1986); "Cosmic Strings and Other Topological Defects", A. Vilenkin and E. P. S. Shellard, Cambridge University Press (1994)

13. E. Chudnovsky and A. Vilenkin, Phys. Rev. Lett. 61, 1043 (1988)

14. http://smsc.cnes.fr/SPI

15. D. Hooper et al., Phys. Rev. Lett. 93, 161302 (2004)

16. G. D. Starkman and T. Vachaspati, Phys. Rev. D 53, 6711 (1996)

17. S. Coutu et al., Astropart.Phys. 11, 429-435 (1999); S.W. Barwick et al., Ap. J. 482, L191 (1997) 